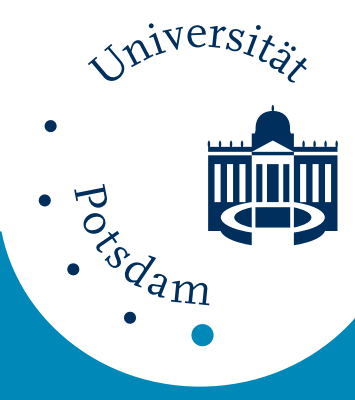

Universität Potsdam

Sten Rüdiger, Fred Feudel, Norbert Seehafer

Dynamo bifurcations in an array of driven convection-like rolls

NLD Preprints ; 43 


\title{
Dynamo bifurcations in an array of driven convection-like rolls
}

\author{
S. Rüdiger, F. Feudel, N. Seehafer \\ Institut für Physik, Universität Potsdam, PF 601553, D-14415 Potsdam, Germany
}

(February 6, 1998)

\begin{abstract}
The bifurcations in a three-dimensional incompressible, electrically conducting fluid with an external forcing of the Roberts type have been studied numerically. The corresponding flow can serve as a model for the convection in the outer core of the Earth and is realized in an ongoing laboratory experiment aimed at demonstrating a dynamo effect. The symmetry group of the problem has been determined and special attention has been paid to symmetry breaking by the bifurcations. The nonmagnetic, steady Roberts flow loses stability to a steady magnetic state, which in turn is subject to secondary bifurcations. The secondary solution branches have been traced until they end up in chaotic states.
\end{abstract}

47.20.Ky, 47.65.+a, 47.27.Cn, 95.30.Qd

\section{INTRODUCTION}

The generation and maintenance of magnetic fields by the motion of electrically conducting fluids as, for example, those in the fluid outer core of the Earth and in the convection zone of the Sun is the subject of dynamo theory. Realistic models, describing the dynamo processes, are given in the form of a complex system of nonlinear partial differential equations including the Navier-Stokes equations (NSE), the induction equation, the heat equation, and the thermodynamic equation of state. Heating causes fluid motions which in turn, notably in the presence of rotation, induce magnetic fields. An account of the relevant equations is, e.g., found in Ref. [1].

Because of the complexity of realistic models and due to restricted computer capacities, which allow only short time simulations, the dynamo processes are not completely understood yet. Looking for tractable models, it is generally accepted that the incompressible magnetohydrodynamic (MHD) equations, consisting of the incompressible NSE and the induction equation, contain the basic elements of a dynamo [2]. Furthermore, traditional dynamo theory has been mainly kinematic, prescribing the velocity field and solving the (then linear) induction equation for the magnetic field, with positive growth rates indicating a dynamo effect. The ABC flow $\boldsymbol{v}_{A B C}$ [3-5] and the Roberts flow $\boldsymbol{v}_{R}[6]$ are intensively studied examples for dynamo-effective velocity fields. To take into account the back reaction of the magnetic field on the velocity field, the kinematic analysis has to be extended to a study of the full nonlinear MHD equations. The necessary energy input into the system may be modeled by an external-forcing term in the NSE which just produces an appropriate velocity field, like $\boldsymbol{v}_{A B C}$ or $\boldsymbol{v}_{R}$, as a solution of the NSE. For small Reynolds numbers, i.e., if the forcing is weak, this velocity field with a vanishing magnetic field is also the only stable (time-asymptotic) solution of the full MHD equations with the introduced external forcing. The dynamo problem can then be reformulated as follows: Does there exist for higher Reynolds numbers a phase transition to solutions with nondecaying magnetic fields? For the case of the $\mathrm{ABC}$ forcing this question could be answered affirmatively [7-10].

In this paper we address the dynamo problem for situations with a forcing of the Roberts type. The Roberts flow has recently received renewed interest. On one hand it resembles the roll solutions of thermal (or solutal) convection. In the convective zones of rotating celestial bodies, for instance, convection rolls parallel to the axis of rotation tend to be formed [11]. On the other hand the Roberts flow is approximately realized in an ongoing experiment aimed at demonstrating the dynamo effect under terrestrial conditions [12]. The experimental setup has been motivated by the kinematic dynamo effectiveness of the Roberts flow (and its supposed resemblance to planetary convection). A conducting fluid (sodium) is pumped through an array of straight parallel ducts which are connected at their ends, where the flow out of a duct reverses its direction before entering a neighboring duct. The ducts contain internal guiding structures such that the flow becomes helical. All guiding structures, including the (thin) walls separating neighboring ducts, are electrically conducting.

Kinematic studies related to this experiment are due to Apel et al. [13] and Tilgner [14]. Apel et al. applied mean-field dynamo theory [15], whose central mechanism is the alpha effect, while Tilgner used direct numerical simulation of the induction equation. In both studies the prescribed flow was the Roberts flow and system parameters most suitable for dynamo excitation were determined. But due to the kinematic nature of the models used the feedback of the magnetic field to the velocity field remained an open problem. This gave us the motivation to study bifurcations of the MHD equations for situation where an external forcing of the Roberts type is applied.

The Roberts flow is given as a family of threedimensional velocity fields which are independent of one of the spatial coordinates, namely,

$$
\boldsymbol{v}_{R}=(g \sin x \cos y,-g \cos x \sin y, 2 f \sin x \sin y),
$$

where $g$ and $f$ are real parameters. This flow is an exact solution of the incompressible Euler equations. It is also a solution of the incompressible NSE if the external force 
field

$$
\boldsymbol{f}=2 \boldsymbol{v}_{R}=-\nabla^{2} \boldsymbol{v}_{R}
$$

is applied, just compensating the viscous losses. Furthermore, together with a vanishing magnetic field, the Roberts flow yields a solution of the the full MHD equations (Eqs. (3-5 below), which is stable for small Reynolds numbers.

In this paper we investigate numerically the bifurcation scenario of the MHD equations with Roberts forcing, the strength of the forcing or the Reynolds number being our bifurcation parameter. We look for magnetic instabilities, which indicate a dynamo effect, and characterize the bifurcating solution branches.

Special attention is paid to symmetry-breaking effects. The first, symmetry-breaking bifurcation is studied by means of equivariant singularity theory [16]. The irreducible representations of the equivariance group determine the possible symmetries of bifurcating solutions. So the subsymmetry of a magnetic branch, bifurcating from the Roberts flow, is both determined analytically and confirmed numerically.

In Sec. II we introduce the governing equations, explain the numerical truncation used, and determine the symmetry group of the equations. Then in Sec. III the bifurcation sequence obtained for increasing the Reynolds number is presented. In Sec. IV we explore the spatial structure of the magnetic field generated in the primary bifurcation and study the symmetry of the total solution (consisting of magnetic and velocity fields). A brief discussion is given in Sec. V.

\section{BASIC EQUATIONS AND SYMMETRY}

We start from the equations for an incompressible electrically conducting fluid, the MHD equations,

$$
\frac{\partial \boldsymbol{v}}{\partial t}+(\boldsymbol{v} \cdot \boldsymbol{\nabla}) \boldsymbol{v}=\nabla^{2} \boldsymbol{v}-\boldsymbol{\nabla} p-\frac{1}{2} \boldsymbol{\nabla} \boldsymbol{B}^{2}+(\boldsymbol{B} \cdot \boldsymbol{\nabla}) \boldsymbol{B}+\boldsymbol{f},
$$

$$
\begin{gathered}
\frac{\partial \boldsymbol{B}}{\partial t}+(\boldsymbol{v} \cdot \boldsymbol{\nabla}) \boldsymbol{B}=P_{m}^{-1} \nabla^{2} \boldsymbol{B}+(\boldsymbol{B} \cdot \boldsymbol{\nabla}) \boldsymbol{v} \\
\boldsymbol{\nabla} \cdot \boldsymbol{v}=0, \quad \boldsymbol{\nabla} \cdot \boldsymbol{B}=0
\end{gathered}
$$

where $\boldsymbol{v}$ denotes the fluid velocity, $\boldsymbol{B}$ the magnetic induction, and $p$ the pressure. Eq. (3) is the NSE, with the Lorentz force and the external force $\boldsymbol{f}$ on the right-hand side, Eq. (4) the induction equation, and the first relation in Eq. (5) the incompressibility condition. We use the MHD equations in a nondimensional form here; the rescaling transformation may be found in Ref. [9]. $P_{m}$ is the magnetic Prandtl number $\left(P_{m}=\nu / \eta\right.$, where $\nu$ is the kinematic viscosity and $\eta$ is the magnetic diffusivity).
Corresponding to the spatial periodicity of the Roberts field given by Eqs. (1) and (2), we apply periodic boundary conditions to the MHD equations and consider their solutions in a cube of side length $2 \pi$. The magnetic Prandtl number is fixed to a value of $1, P_{m}=1$, and the two parameters of the velocity field are set equal to each other, $f \equiv g$. Following previous studies of the ABC forced MHD equations [7-10], the parameter $f=g$ is referred to as the Reynolds number $R$.

To give an impression of the Roberts flow, the only time-asymptotic state for small $R$, projections of the velocity vectors on the $x-y$ plane are shown in Fig. 1 (left). The velocity field splits up into separate cells where the streamlines spiral up or down. It is a prevalent conjecture that the presence of kinetic helicity, defined by

$$
H_{k}=\boldsymbol{v} \cdot \boldsymbol{\nabla} \times \boldsymbol{v},
$$

is favourable for a dynamo effect. In Fig. 1 (right) therefore also contour lines of the kinetic helicity are shown.
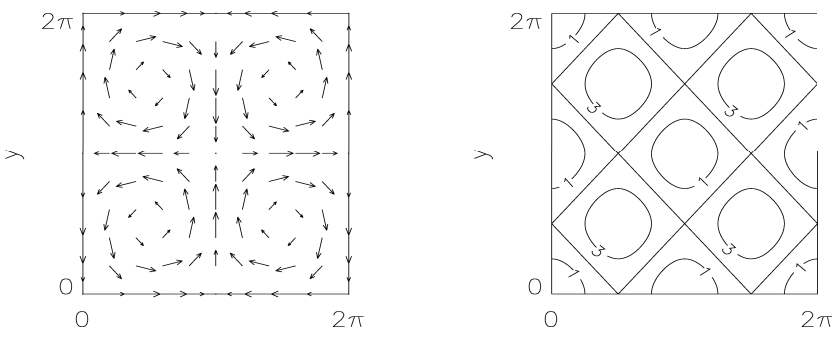

FIG. 1. Projections of the velocity vectors of the Roberts flow on the $x-y$ plane (left) and contour lines of the kinetic helicity in the $x-y$ plane (right). The flow goes up in the lower left and the upper right quadrant. Helicity is largest in the rolls.

In general, an external forcing reduces the symmmetry of the MHD equations. In our case of the Roberts forcing we identify a symmetry group which is isomorphic to the group $D_{4} \times Z_{2} \times S^{1} . D_{4}$ denotes the dihedral group. It consists of all rotations and reflections of a square in a plane that leave the square invariant. $Z_{2}$ is the reflection group and corresponds to the translation $(x, y, z) \rightarrow(x+\pi, y+\pi, z)$, that is, to the translation of one roll to that one with the same direction of the fluid motion. $S^{1}$, finally, denotes the circle group corresponding to the translation invariance of the Roberts pattern along the $z$ axis. Of course the primary solution, the Roberts flow, shares these symmetries.

For the numerical calculations we have used Fourier decompositions of $\boldsymbol{v}, \boldsymbol{B}, p$, and $\boldsymbol{f}$ to approximate the MHD equations by a high-dimensional system of ordinary differential equations (ODEs), which then has been treated by means of a pseudospectral method. The mean values of $\boldsymbol{v}$ and $\boldsymbol{B}$ over the cube, which can be shown to be time-independent, have been assumed to vanish. Time integration was performed using an eigth-order Runge- 
Kutta scheme with adaptive time-stepping and the pseudospectral code was used with a spatial resolution of $16^{3}$ collocation points, corresponding to a system of 49152 ODEs; by employing Eq. (5) this number of equations is reduced by one third. The final system of ODEs has been studied by means of simulations and bifurcation-analysis techniques.

\section{BIFURCATIONS AND ATTRACTORS}

For small $R$ the Roberts flow with a vanishing magnetic field is the only stable solution of the MHD equations. Tracing this solution branch to higher Reynolds numbers we have calculated the eigenvalues of the Jacobian to look for bifurcations. At $R \approx 7.3$ two real eigenvalues, which belong to magnetic modes, become equal to zero. This dynamo instability yields a symmetry breaking pitchfork bifurcation generating new steady states with a nonvanishing magnetic field. The original symmetry is broken, namely, the solutions are no longer $S^{1}$ invariant; now both the magnetic field and the velocity field have got a dependence on the $z$ coordinate. This reduces the symmetry of the solutions to the subgroup symmetry $D_{4} \times Z_{2}$. A more detailed discussion of this symmetry breaking bifurcation by means of representation theory is given in Sec. IV. The generated magnetic field acts on the fluid motion. But though now nearly all Fourier modes of the velocity fields are excited, the original modes of the Roberts flow still dominate the structure of the velocity field.

Time averages of the magnetic energy versus the Reynolds are shown in Fig. 2, where also the different attractor types are indicated. The onset of a dynamo at $R \approx 7.3$ is clearly seen. For increasing Reynolds numbers the magnetic energy grows strongly along the stationary magnetic branch, which ends up in a secondary bifurcation at $R \approx 12.5$ generating time periodic solutions. The resulting structures of the velocity field are wavy rolls: The fluid still moves in four seperate rolls, as depicted in Fig. 1, but these are now weakly bended and oscillate slightly.

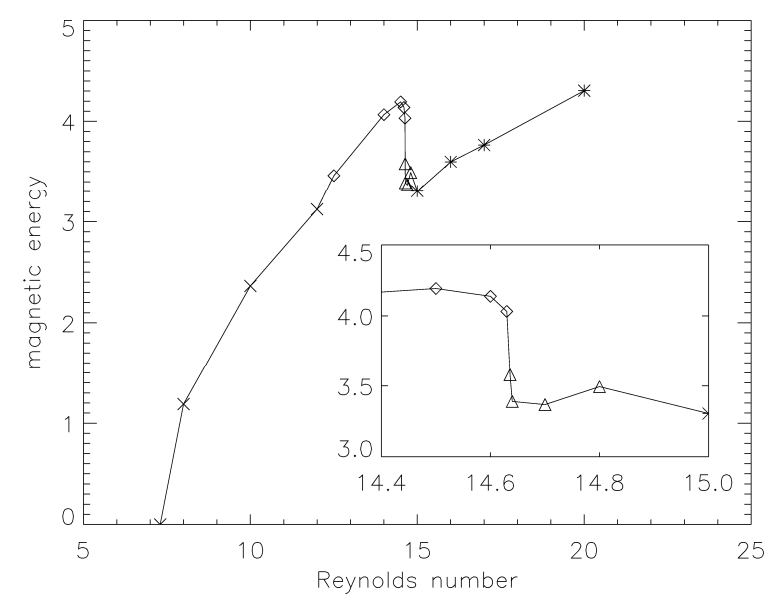

FIG. 2. Magnetic energy versus Reynolds number. Steady-state $(\times)$, periodic $(\diamond)$, torus $(\triangle)$, and chaotic solutions $(*)$ are marked. The inner small box shows the zoomed region for $R=14.4 \ldots 15.0$.

In Fig. 2 the sudden drop of the magnetic energy at $R \approx 14.65$ leaps to the eyes. It is accompanied by a transition from the periodic branch to quasiperiodic solutions (torus branch). We resolved the transition region with high accuracy, as can be seen in the zoomed part of Fig. 2, but we could not clarify in detail the origin of the torus branch. There are at least two possibilities: Both branches are linked by a secondary Hopf bifurcation or they are completely disconnected.

In order to understand the sudden drop of the magnetic energy, we have also studied the dynamics in the purely hydrodynamical case (with no magnetic field). In simulations of the NSE we observed a drop of the kinetic energy. Fig. 3 shows the kinetic energy for both the MHD and the purely hydrodynamic situations. The solid line refers to the MHD equations and the dotted line to the NSE. Surprisingly, the sudden drop of the magnetic energy has nearly no effect on the velocity field. Only a small hump in the vicinity of the instability is visible. We even cannot give a sound interpretation of this hump. It might be an artifact of the averaging procedure used to calculate the kinetic energy (namely, the new, second frequency of the arising torus solution is extremely small). The kinetic energy in the purely hydrodynamic situation shows nearly the same profile as does the magnetic energy in the MHD case (Fig. 2), with the exception that the instability point is shifted a bit towards smaller Reynolds numbers $(R \approx 12)$. This shift can be explained by the additional dissipation in the presence of a magnetic field (so that the forcing must be stronger for the instability to appear). Thus, we are led to conclude that the decrease of the magnetic energy seen in Fig. 2 is primarily caused by a purely hydrodynamic instability. Seemingly the instability reduces the dynamo effectiveness of the velocity field and the associated reduction of the energy transfer from the velocity field to the magnetic field compensates the kinetic-energy drop seen in the purely hydrodynamic 
situation.

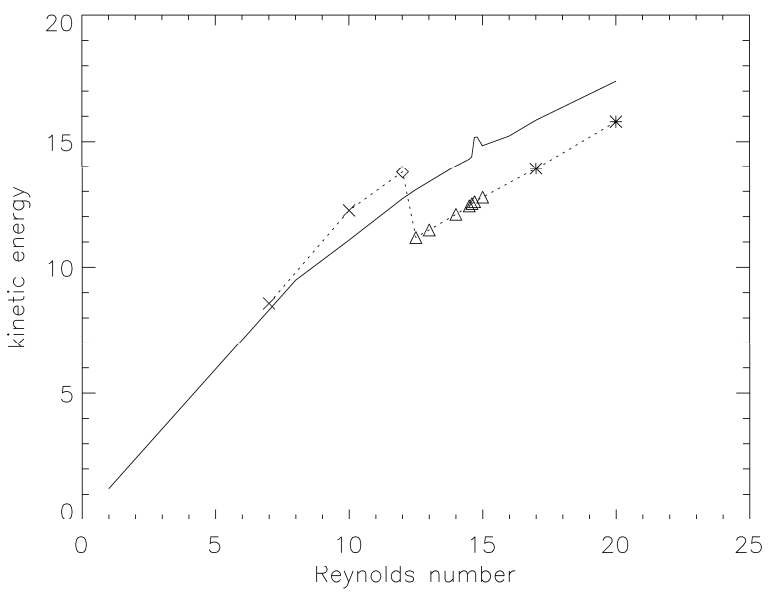

FIG. 3. Kinetic energy versus Reynolds number. The solid line refers to the MHD case and the dotted line to the purely hydrodynamical case. Steady-state $(x)$, periodic $(\diamond)$, torus $(\triangle)$, and chaotic solutions $(*)$ are marked.

The time evolution of a trajectory for $R=14.7$, projected onto a plane in phase space, is drawn in Fig. 4. As it can be seen from the zoomed part of Fig. 2, this quasiperiodic branch is stable only within a small interval of the Reynolds number and loses stability between $R=14.8$ and $R=15.0$. For larger values of the Reynolds number chaos is observed. In Fig. 5 we present an example of a chaotic trajectory for $R=17.0$.

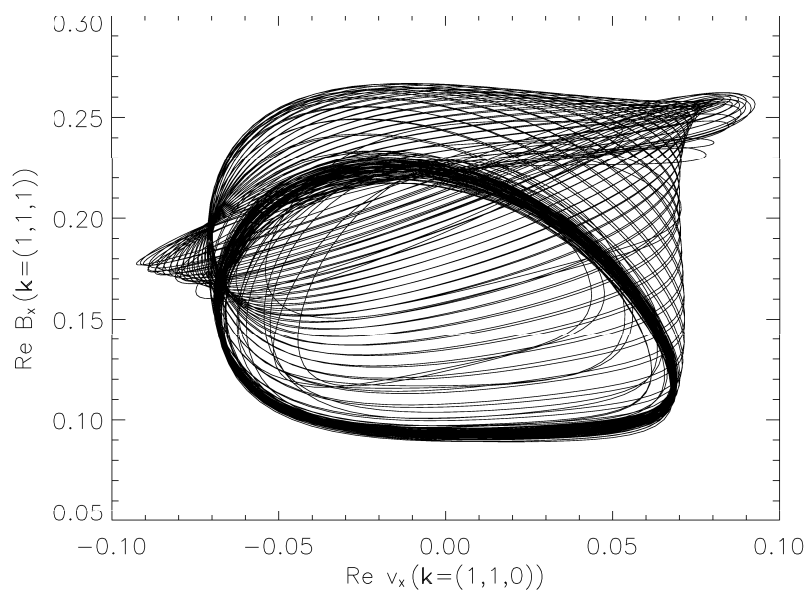

FIG. 4. Projection of the torus solution onto a plane spanned by one of the velocity and one of the magnetic-field Fourier components for $R=14.7$.

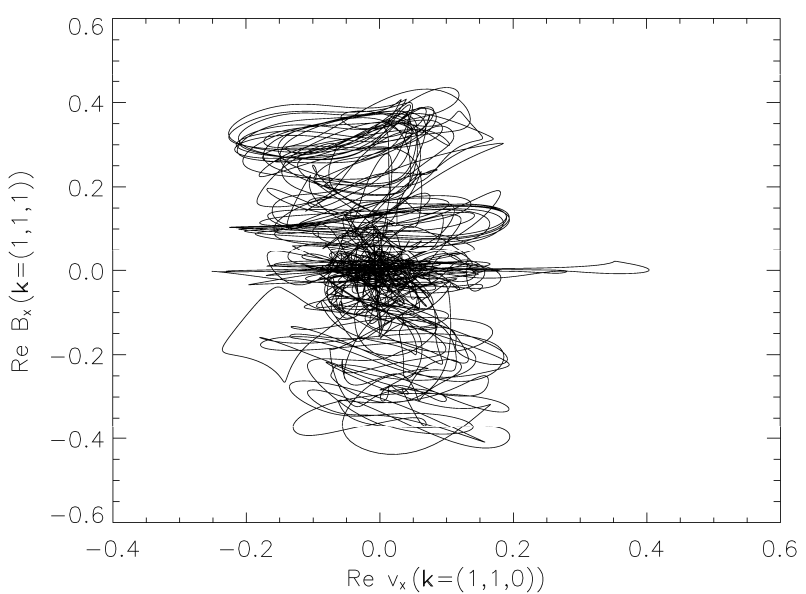

FIG. 5. Projection of the chaotic solution onto a plane spanned by one of the velocity and one of the magnetic-field Fourier components for $R=15.0$.

To prove the chaoticity we have calculated Lyapunov exponents, using an algorithm of Shimada and $\mathrm{Na}$ gashima [17]. To check the correctness of the algorithm, we applied the program, firstly, to the secondary steady state at $R=7.3$. As expected, one Lyapunov exponent was found to be equal to zero, corresponding to the marginally stable direction which results from the breaking of the $S^{1}$ symmetry. This vanishing Lyapunov exponent remains also present for the following branches. Thus, the periodic and the torus solutions possess two or three vanishing exponents, respectively. For the chaotic solution at $R=15.0$ we found at least two positive Lyapunov exponents. Fig. 6, showing the cumulative value of the five largest Lyapunov exponents as a function of the integration time, demonstrates the good convergence of the algorithm.

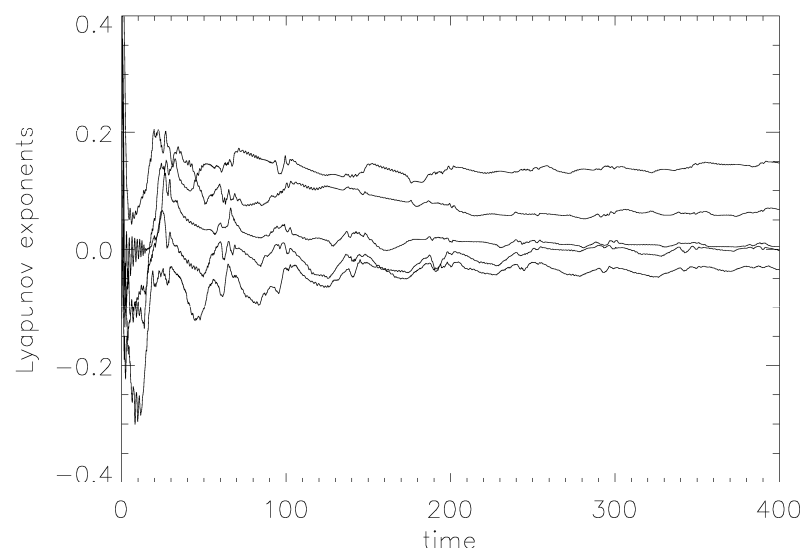

FIG. 6. The five largest Lyapunov exponents versus integration time for $R=17$. 


\section{STRUCTURE OF THE MAGNETIC FIELD}

In this section we characterize the real-space structure of the magnetic field on the stationary magnetic branch and for the chaotic regime.

In the symmetry breaking bifurcation at $R \approx 7.3$, which produces the secondary steady state solution with a nonvanishing magnetic field, two real eigenvalues of the Jacobian matrix become equal to zero. This symmetry breaking bifurcation will be discussed in the frame of group representation theory. We will not present a complete proof but rather sketch only its idea.

Fourier space can be decomposed into isotypic components which correspond to the irreducible representations of the symmetry group. Since two real eigenvalues become equal to zero, a two-dimensional irreducible representation of the original symmetry group $D_{4} \times Z_{2} \times S^{1}$ has been studied. Considering the complex plane, the natural action of $D_{4}$ is generated by the rotation $\gamma=\exp (\imath \pi / 2)$ and the reflection $\kappa$ in the real axis. Let the translation $Z_{2}$ act as the identity and $s \in S^{1}$ as a multiplication with $\exp (\imath s)$. This is also the irreducible representation which we have found to be responsible for the two vanishing eigenvalues.

It turns out that the corresponding eigenvectors lie in a subspace of Fourier space spanned by the Fourier modes $\exp \left(\imath k_{x} x+\imath k_{y} y+\imath k_{z} z\right)$ with $k_{z}=-1,1$ and $k_{x}+k_{y}$ even. It can be shown that this subspace decomposes into finite-dimensional components which are invariant with respect to the action of the symmetry group. This results from the action of the symmetry group elements on the $\boldsymbol{k}$ vectors of the Fourier modes, which conjugate the elements of certain subsets of the $\boldsymbol{k}$ vectors. For instance, the vectors $(1,0,0),(0,1,0),(-1,0,0),(0,-1,0)$ are mapped to each other by the action of the symmetry elements in real space. These finite dimensional subspaces are reducible and can be decomposed into their irreducible components. It can be shown from the action of the symmetries in Fourier space that the representation described above occurs in the decomposition of each of these subspaces, i.e., in each subspace there is at least one two-dimensional subspace in which the symmetry group acts as this representation.

Employing the equivariant branching theorem [16] it can be proved that for this representation at least one branch of solutions with a "twisted" symmetry group isomorphic to $D_{4} \times Z_{2}$ bifurcates. This symmetry group $\Sigma$ is generated by $\left\{(\gamma,-\gamma),(\kappa, i d) \in D_{4} \times S^{1}\right\} \times Z_{2}$. The translational symmetry $S^{1}$ is broken. Due to the $S^{1}$ symmetry for every $s \in S^{1}$ there exists a solution with symmetry group $\widehat{\Sigma}^{s}=s \Sigma s^{-1}$ where $\Sigma$ is the described generated symmetry. Thus, we obtain a family of solutions which are translated in the $z$ direction with isomorphic symmetry groups.

In Fig. 7 we have plotted the absolute value of the magnetic field in the cross sections $z=\pi / 2$ and $z=\pi$ for $R=8$, i.e., for a solution on the secondary steady-state branch. This solution is symmetric with respect to one of the isomorphic groups $\hat{\Sigma}^{s}$. Since in Fig. 7 only the contour lines of the magnetic field strength are drawn, some symmetry properties of the three-dimensional vector field are not visible, but certain symmetry properties are obvious; we only mention the translational symmetry which corresponds to the $Z_{2}$ component of the original group and the rotational symmetry around the $y$-axis.

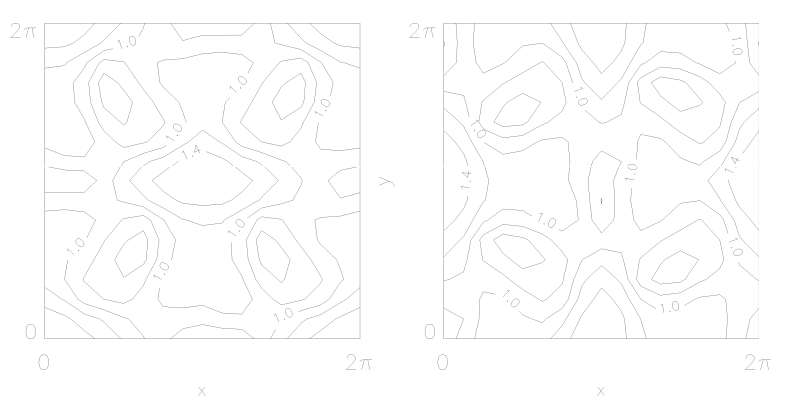

FIG. 7. Contour lines of the absolute value of the magnetic field in the planes $z=\pi / 2$ (left) and $z=\pi$ (right) for $R=8$.

Three-dimensional plots show that there are cylinders of weak magnetic field in the interiors of the fluid rolls. By comparison with Fig. 1 one can see that they coincide with the regions of largest helicity. This phenomenon seems to be a result of flux expulsion from centers of helical motions (cf., e.g., Ref. [2]). "Lumps" of strong magnetic field are situated between the weak-field regions (see Fig. 7), the locations of the field maxima coinciding approximately with the stagnation points (or lines, respectively) of the original Roberts flow. The strong fields here are probably due to the combined effects of flux expulsion from the rolls and field-line stretching near the stagnation points.

The symmetry of the secondary steady state implies that the modes with wavenumbers $\boldsymbol{k}=\left(k_{x}, 0,0\right)$ and $\boldsymbol{k}=$ $\left(0, k_{y}, 0\right), k_{x}, k_{y} \in \mathbb{N}$, have to be equal to zero. After the Hopf bifurcation (leading to the time-periodic state) all these modes are excited. Those with $k_{x}=1$ or $k_{y}=1$, in particular, represent large-scale shear components of the flow and of the magnetic field.

For the chaotic regime all symmetries seem to be broken. To give an impression of the irregular structure of the magnetic field in this regime, contour lines of the absolute value of the magnetic field in two cross sections through the cube, calculated for $R=17$, are shown in Fig. 8. 


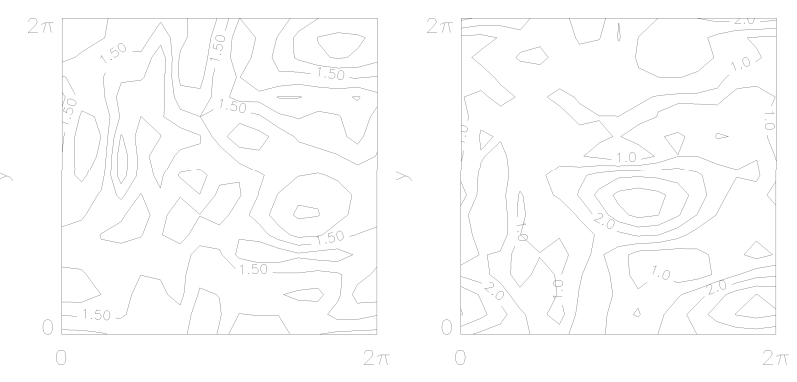

FIG. 8. Contour lines of the absolute value of the magnetic field in the planes $z=\pi / 2$ (left) and $z=\pi$ (right) for $R=17$.

To get a measure of the degree of spatial irregularity in the chaotic state, also energy spectra have been calculated. Compared to the periodic state depicted in Fig. 7, where only one mode with a nonvanishing $k_{z}$ is excited, the $k_{z}$ spectra of the chaotic solutions extend to smaller scales. Here the $k_{z}$ spectra are calculated by averaging over all $k_{x}$ and $k_{y}$. In the $|\boldsymbol{k}|$ spectrum, however, we could not observe yet (for the Reynolds numbers considered) significant differences between the chaotic and other time-dependent solution branches.

\section{SUMMARY}

We have studied the incompressible MHD equations with a forcing of the Roberts type. For increasing the Reynolds number from small values, the primary solution, the nonmagnetic Roberts flow, becomes unstable in a pitchfork bifurcation yielding a new steady state with a nonvanishing magnetic field, that is to say, a dynamo effect. In contrast to analogous investigations of the MHD equations with $\mathrm{ABC}$ forcing, where the primary dynamo bifurcation is of the Hopf type and all magnetic states are time-dependent [7-9], it seems remarkable that a stationary dynamo has been found here.

We have traced the magnetic solution branch towards higher Reynolds number and have found a transition to chaotic solutions via a series of secondary bifurcations.

A notable detail in the bifurcation sequence is a sudden drop of the magnetic energy accompanying the transition from periodic to quasiperiodic motion. It is likely to be caused by a primarily hydrodynamic instability. While in the purely hydrodynamic case a kinetic-energy drop is observed, there is a magnetic-energy drop (and no kinetic-energy drop) in the MHD case.

Special attention has been paid to the analysis of the first symmetry-breaking bifurcation and its influence on the structure of the generated magnetic field. We have determined the magnetic modes becoming unstable in the primary pitchfork bifurcation and have classified the resulting subgroup which determines the symmetry of the new steady state with a nonvanishing magnetic field; it turned out that the original translational symmetry is broken in a nontrivial manner.

\section{ACKNOWLEDGMENTS}

This work was supported by the Deutsche Forschungsgemeinschaft under its main topic "Ergodentheorie, Analysis und effiziente Simulation dynamischer Systeme".

[1] E. R. Priest, Solar Magnetohydrodynamics (D. Reidel Publishing Company, Dordrecht, 1982).

[2] H. K. Moffatt, Magnetic Field Generation in Electrically Conducting Fluids (Cambridge University Press, Cambridge, England, 1978).

[3] V. I. Arnold, C. R. Acad. Sci. Paris 261, 17 (1965).

[4] V. I. Arnold and E. I. Korkina, Vest. Mosk. Univ. Mat. Mekh. 3, 43 (1983).

[5] D. Galloway and U. Frisch, Geophys. Astrophys. Fluid Dyn. 36, 53 (1986).

[6] G. O. Roberts, Phil. Trans. Roy. Soc. London A 271, 411 (1972).

[7] B. Galanti, P. L. Sulem, and A. Pouquet, Geophys. Astrophys. Fluid Dyn. 66, 183 (1992).

[8] N. Seehafer, F. Feudel, and O. Schmidtmann, Astron. Astrophys. 314, 693 (1996).

[9] F. Feudel, N. Seehafer, B. Galanti, and S. Rüdiger, Phys. Rev. E 54, 2589 (1996).

[10] F. Feudel, N. Seehafer, and O. Schmidtmann, Phys. Lett. A 202, 73 (1995).

[11] F. H. Busse, Chaos 4, 123 (1994).

[12] R. Stieglitz and U. Müller, GEODYNAMO - Eine Versuchsanlage zum Nachweis des homogenen Dynamoeffektes, Research Report FZKA-5716, Forschungszentrum Karlsruhe (1996).

[13] A. Apel, E. Apstein, K.-H. Rädler, and M. Rheinhardt, Contributions to the theory of the Karlsruhe dynamo experiment, Research Report, Astrophysikalisches Institut Potsdam (1996).

[14] A. Tilgner, Phys. Lett. A 226, 75 (1997).

[15] F. Krause and K.-H. Rädler, Mean-Field Magnetohydrodynamics and Dynamo Theory (Akademie-Verlag, Berlin, 1980).

[16] M. Golubitsky, I. Stewart, and D. G. Schaeffer, Singularities and Groups in Bifurcation Theory (Springer, New York, 1988), Vol. II.

[17] I. Shimada and T. Nagashima, Progr. Theor. Phys. 61, 1605 (1979). 